\title{
Looming Biases in Monkey Auditory Cortex
}

\author{
Joost X. Maier and Asif A. Ghazanfar \\ ${ }^{1}$ Max Planck Institute for Biological Cybernetics, 72076 Tuebingen, Germany
}

Looming signals (signals that indicate the rapid approach of objects) are behaviorally relevant signals for all animals. Accordingly, studies in primates (including humans) reveal attentional biases for detecting and responding to looming versus receding signals in both the auditory and visual domains. We investigated the neural representation of these dynamic signals in the lateral belt auditory cortex of rhesus monkeys. By recording local field potential and multiunit spiking activity while the subjects were presented with auditory looming and receding signals, we show here that auditory cortical activity was biased in magnitude toward looming versus receding stimuli. This directional preference was not attributable to the absolute intensity of the sounds nor can it be attributed to simple adaptation, because white noise stimuli with identical amplitude envelopes did not elicit the same pattern of responses. This asymmetrical representation of looming versus receding sounds in the lateral belt auditory cortex suggests that it is an important node in the neural network correlate of looming perception.

Key words: lateral belt; time to collision; time to contact; gamma band; auditory motion; local field potential

\section{Introduction}

Looming signals are those sensory cues that indicate the rapid approach of objects. Because of their relevance for survival, it is thought that animals have an evolved bias for detecting and responding to looming events (Schiff et al., 1962; Neuhoff, 2001; Ghazanfar et al., 2002; Maier et al., 2004). Looming is well studied in the visual domain, and species across the animal kingdom show a behavioral bias for detecting and responding to visual looming, compared with receding signals (Schiff, 1965). For example, Schiff et al. (1962) showed that rhesus monkeys respond to visual looming stimuli by making defensive movements and producing alarm calls. In some species, adaptive responses to visual looming signals are mediated by specialized neural circuitry (Sun and Frost, 1998; Gabbiani et al., 2002).

In the auditory domain, looming signals are characterized by specific intensity and temporal parameters. As a sound source approaches/recedes, intensity at the ears of the observer rises/ falls. Although other cues, like interaural and reverberatory cues, can also be important for localizing moving sound sources, dynamic intensity change is the dominant and most effective cue for detecting sound source motion in depth (Rosenblum et al., 1987; Zakarauskas and Cynader, 1991; Lufti and Wang, 1999). Thus, looming sound sources are best characterized by rising intensity sounds and receding sources by falling-intensity sounds, and looming events take place at time scales from hundreds of milliseconds to seconds.

Received Jan. 24, 2007; revised March 5, 2007; accepted March 7, 2007.

This work was supported by the Max Planck Society. This study was conducted under the auspices of Dr. Nikos Logothetis. Without his encouragement and generosity, this work would not have been possible. We thank Kari Hoffman for her constructive support during the study, Hjalmar Turesson for help with the recordings, and Hjalmar and Chandramouli Chandrasekaran for their comments on this manuscript.

Correspondence should be addressed to Asif A. Ghazanfar, Program in Neuroscience, Department of Psychology, Green Hall, Princeton University, Princeton, NJ 08540. E-mail: asifg@princeton.edu.

DOI:10.1523/JNEUROSCI.0330-07.2007

Copyright $\odot 2007$ Society for Neuroscience $\quad$ 0270-6474/07/274093-08\$15.00/0
Auditory looming signals also reveal perceptual biases in observers. Human adults and infants (Small, 1977; Morrongiello et al., 1991; Reinhardt-Rutland, 1996b; Neuhoff, 1998; Freiberg et al., 2001) and rhesus monkeys (Ghazanfar et al., 2002) hear simulated looming sounds as more salient than receding ones. The increased saliency is evident in increased attention toward looming sounds, estimation of time to arrival, and/or estimation of loudness change. For example, when subjects are required to use dynamic intensity cues to indicate the time to arrival of a looming sound source, they consistently err on the side of safety (Rosenblum et al., 1987; Schiff and Oldak, 1990; Neuhoff, 2001). Importantly, these perceptual biases are only evident when the sounds have some spectral structure; rising/falling-intensity white noise stimuli do not elicit the percepts of looming/receding and do not reveal any perceptual biases (Neuhoff, 1998; Ghazanfar et al., 2002; Maier et al., 2004).

Most neurophysiological studies investigating spatial processing in the auditory cortex focus on responses to short, static noise sounds (Middlebrooks and Pettigrew, 1981; Recanzone et al., 2000b), but, as the case of looming shows, salient signals are often dynamic and spectrally structured. Furthermore, a given animal's auditory system is likely adapted to respond to behaviorally relevant, natural features of sound sources (Nelken et al., 1999; Dean et al., 2005; Garcia-Lazaro et al., 2006). Here, by mimicking looming and receding signals with rising- and falling-intensity complex tones, we explore the neural processing of these dynamic, highly salient auditory signals in the auditory cortex of the rhesus monkey. Based on the perceptual biases described above, we hypothesize that looming sounds are processed with priority at the neural level as well.

\section{Materials and Methods}

Subjects. Two adult male rhesus monkeys (Macaca mulatta) were used as subjects in the experiments. For each subject, we used preoperative magnetic resonance imaging ( $4.7 \mathrm{~T}$ magnet; $500 \mu \mathrm{m}$ slices) to identify the stereotaxic coordinates of auditory cortex and to model a three- 
dimensional skull reconstruction. From these skull models, we constructed custom-designed, form-fitting titanium headposts and recording chambers. The subjects underwent sterile surgery for the implantation of a scleral search coil (Robinson, 1963), headpost, and recording chamber. The inner diameter of the recording chamber was $19 \mathrm{~mm}$ and was vertically oriented to allow a perpendicular approach to the surface of the superior temporal plane (Pfingst and O'Connor, 1980; Recanzone et al., 2000a). All experiments were performed in compliance with the guidelines of the local authorities (Regierungspraesidium) and the European Community (EU VD 86/609/EEC) for the care and use of laboratory animals.

Stimuli. Auditory stimuli, rising- and fallingintensity complex tones and white noise, were generated with SoundForge (Sonic Foundry, Madison, WI) and Adobe Audition (Adobe Systems, San Jose, CA) (Fig. $1 A$ ). Hereafter, we will refer to the rising-intensity complex tones as the "looming" signal and the falling-intensity complex tones as the "receding" signals, because this cue alone seems to elicit the corresponding percepts (Rosenblum et al., 1987; Neuhoff, 2001). The amplitude envelopes for all stimuli either rose or fell quadratically over a period of $1000 \mathrm{~ms}$. Figure 1, $C$ and $D$, shows the frequency spectra of the complex tones (fundamental frequency, $1000 \mathrm{~Hz}$ ) and white noise, respectively. All stimuli started and ended with a $300 \mathrm{~ms}$ static period flanking the dynamic interval, resulting in stimuli with a total duration of $1600 \mathrm{~ms}$. To control for differences in absolute intensity between looming and receding stimuli, initial intensity was set at two different levels: from 68 to $86 \mathrm{~dB}$ sound pressure level (SPL) for the "loud" stimuli and 50-68 dB SPL for the "soft" stimuli, as measured with a Brüel \& Kjær (Norcross, GA) sound level meter (A-weighted) at a distance of $94 \mathrm{~cm}$. Critically, the overall intensity of the soft looming stimulus was always lower than that of the loud receding stimulus. This stimulus set allowed us to differentiate between neural responses that were more dependent on the absolute intensity (loud vs soft) of the sound and neural responses that were more dependent on the direction of intensity change (looming vs receding). Complex tone and white noise stimuli had equal average root-meansquare energy.

Behavioral paradigm. Experiments were conducted in a double-walled sound-attenuating booth, lined with echo-attenuating foam. The subjects were head restrained and sat in a primate chair in front of a 21 inch color monitor at a distance of $94 \mathrm{~cm}$. On both sides of the monitor, a speaker was placed (Control 1X; JBL, Northridge, CA). Subjects performed in the dark, and a trial began with the appearance of a central fixation spot. Subjects were required to fixate this spot within a 1 or $2^{\circ}$ radius. After $500 \mathrm{~ms}$ of fixation, an auditory stimulus was presented for $1600 \mathrm{~ms}$. Subjects had to maintain fixation throughout the duration of the stimulus. Successful completion of a trial resulted in a juice reward. During each recording session, between 10 and 20 repetitions of each stimulus were presented.

Data collection. All events relevant to the experiment, such as stimulus information and eye position, were stored. Eye position was sampled at $200 \mathrm{~Hz}$. For recording of neural events, we used a custom-made multielectrode drive that allowed us to move up to eight electrodes independently. Electrodes were arranged in a $4 \times 2$ staggered array, covering 12 $\mathrm{mm}$ in the anterior-posterior dimension and $1.5 \mathrm{~mm}$ in the mediallateral dimension. Guide tubes ( 25 gauge) were used to penetrate the tissue growth and dura overlying the cortex. Electrodes were glass-coated tungsten wire with impedances between 1 and $3 \mathrm{M} \Omega$ measured at $1 \mathrm{kHz}$ (Alpha Omega Engineering, Nazareth, Israel). Signals from the electrodes were amplified, filtered between 1 and $5000 \mathrm{~Hz}$, acquired with a sampling rate of $20.8 \mathrm{kHz}$, and stored for off-line analysis [amplification and filtering, Alpha Omega Engineering; acquisition via custom designed software and a National Instruments (Austin, TX) board, BNC-2090].

Identification of auditory cortex. In the current study, we focused on the lateral belt cortex. Electrodes were lowered until multiunit activity (MUA) could be reliably driven by auditory stimuli. Search stimuli included pure tones, frequency-modulated sweeps, noise bursts, clicks, and monkey vocalizations. For each recording site, we made frequency tuning curves using the MUA responses to 25 pure tone pips ( $100 \mathrm{~Hz}$ to 21 $\mathrm{kHz}$ ) presented at an intensity of $72 \mathrm{~dB}$. In our initial mapping of the recording chamber, we discerned a coarse tonotopic map representing high-to-low frequencies in the caudal-to-rostral direction. Such a map is identified as primary auditory cortex (A1). When the electrode array was moved $2 \mathrm{~mm}$ in the lateral direction, the same rough tonotopic map was observed, but complex stimuli (noise, clicks, and vocalizations) generally elicited much stronger responses than pure tones, indicating that the electrodes were in lateral belt auditory cortex (Rauschecker et al., 1995; Recanzone et al., 2000a; Barbour and Wang, 2003; Rauschecker and Tian, 2004) adjacent to A1 [the middle lateral belt area (Hackett et al., 1998)]. The core and lateral belt regions are also distinguished by their sensitivity to multisensory inputs (Ghazanfar et al., 2005; Lehmann et al., 2006). These physiological criteria serve only as a rough guide, and it is likely that some of our electrodes were occasionally placed in rostrally adjacent regions of the lateral belt.

Data analysis. Signals were recorded continuously and processed separately to obtain local field potential (LFP) activity and MUA. All data analyses were performed in Matlab (Mathworks, Natick, MA). To obtain LFP activity, the raw signal was filtered between 1 and $300 \mathrm{~Hz}$ (fourth order, zero-phase, bidirectional Butterworth filter) and resampled at $1000 \mathrm{~Hz}$. To extract spiking activity, the raw signal was loaded into an off-line spike-sorting program (Plexon, Dallas, TX) and high-pass filtered at $521 \mathrm{~Hz}$. MUA was defined as all spike waveforms exceeding the noise level by 4 SD units. Spikes were binned at $200 \mathrm{~Hz}$ for analysis. Spike density functions were created for display purposes only by convolving the raw spike times with a $10 \mathrm{~ms}$ Gaussian kernel.

For LFP analyses, we applied multitaper spectral analysis to estimate 
spectral power (Mitra and Pesaran, 1999; Pesaran et al., 2002) using the Chronux suite of routines developed in Matlab for neural analyses (www. chronux.org). For time-frequency analysis of spectral power, we used nine Slepian data tapers on a $250 \mathrm{~ms}$ sliding window (shifted by $50 \mathrm{~ms}$ ) (Pesaran et al., 2002; Palanca and DeAngelis, 2005; Scherberger et al., 2005). To extract LFP time courses, power was averaged over frequencies of interest (Raghavachari et al., 2001). All data were normalized to baseline before averaging over trials/recording sites. A looming preference index was then calculated by subtracting neural activity in response to the dynamic period of the receding stimuli from the activity in response to the dynamic period of the looming stimuli.

\section{Results}

We recorded LFP and MUA from a total of 50 cortical sites in the lateral belt region of auditory cortex in two monkeys fixating centrally while listening to looming and receding sounds (33 cortical sites in subject $1 ; 17$ in subject 2). Recordings were made from lateral belt cortex because, compared with core areas, neurons in this region respond better to complex sounds and have larger receptive fields (Morel et al., 1993; Rauschecker et al., 1995; Recanzone et al., 2000a). The main focus of the study is on the modulation of power in the LFP signal. LFP power was measured using multitaper spectral estimation. There are two reasons for focusing on the LFP signal. First, we were interested in the responses to the dynamic portion of our stimuli, and therefore we had to exclude the transient onset and offset responses to the stimuli (Fig. 2A). The single neuron responses in monkey auditory cortex have many response profiles (Recanzone, 2000), but, unless the preferred stimulus is identified (Wang et al., 2005), responses tend to be transient. Thus, the sustained, yet selective, sensory responses of LFP signals make them ideal in the current context (Brosch et al., 2002). Second, our stimulus parameters were fixed, and recording sites were selected randomly to get an unbiased estimate of the neural sensitivity of lateral belt auditory cortex to looming/receding sounds. Naturally, this limits the chances of finding single units that are optimally tuned to the stimuli. However, we did measure MUA responses as a coarse estimate of unit responses to our stimuli. Multiunit activity, like the LFP, is available for analyses without specifically selecting neurons, which would bias the sample of the neuronal population (Super and Roelfsema, 2005).

\section{Looming versus receding sounds: LFP activity}

To clearly differentiate responses to looming versus receding sounds, we had to eliminate absolute intensity as a factor. To do this, we used two different levels of looming and receding sounds: a loud version and a soft version. Importantly, the "looming soft" condition is lower in overall intensity relative to the "receding loud" condition, and the average intensity of the looming conditions (loud + soft) is equal to the average intensity of the receding conditions (Fig. $1 B$ ). Figure $2 A$ shows the raw LFP activity from a single cortical site in response to the combined looming (loud + soft) and receding (loud + soft) sounds. To be able to separate the transient onset and offset responses from the response to the dynamic portion of the sound, the dynamic portion of the stimulus was preceded and followed by a $300 \mathrm{~ms}$ static period (Fig. $1 B$ ). Onset and offset of the auditory stimuli are marked by dashed vertical lines. These portions of the auditory stimuli elicited phasic onset and offset responses. However, the activity of interest in the present study is the activity in response to the 1000 $\mathrm{ms}$ dynamic portion (change in intensity) of the sound, marked by the solid vertical lines, and all analyses are based on activity taken from this period. As can been seen from Figure $2 \mathrm{~A}$, the amplitude of the raw LFP was not differentially modulated dur-
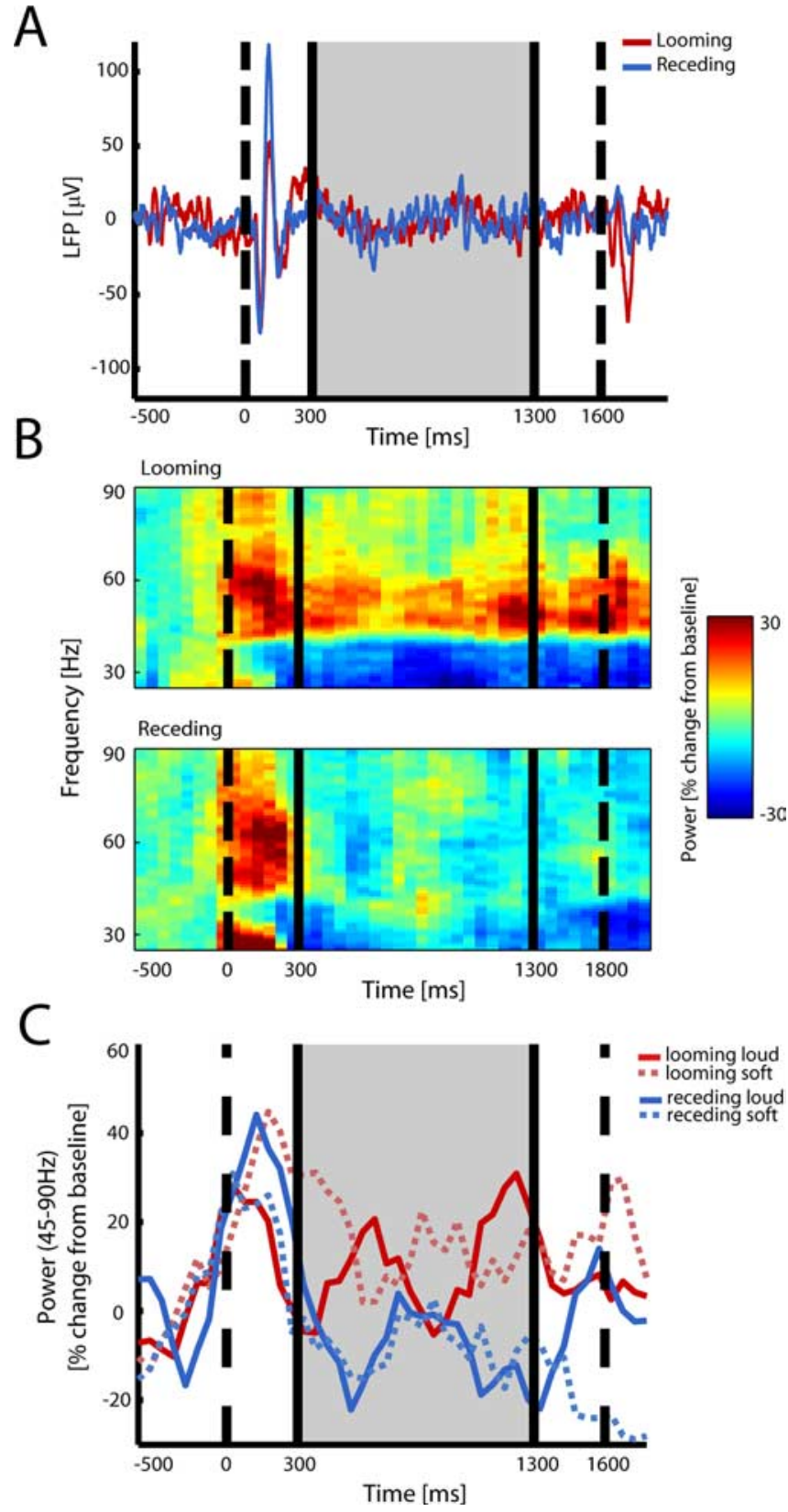

Figure 2. A, Example raw local field potential responses to looming and receding stimuli in a single cortical site. Shown is a time-amplitude representation of the LFP signal recorded from one example cortical site in response to looming (red) and receding (blue) stimuli. Dashed vertical lines represent onset and offset of the auditory stimuli. Solid vertical lines mark the start and end of the dynamic portion of the stimuli. Traces represent the mean response over 36 trials per condition. $\boldsymbol{B}$, Example spectrograms of the LFP response to looming and receding stimuli. Shown are power changes over time and frequency, relative to baseline, in response to looming and receding stimuli. Spectrograms represent the mean response over 36 trials per condition (same site as in $\boldsymbol{A}$ ); that is, loud and soft stimuli are averaged together. C, Example gamma-band power in the LFP in response to loud and soft looming and receding stimuli. Shown is gammaband $(45-90 \mathrm{~Hz})$ power in the LFP over time, relative to baseline, in response to loud (solid lines) and soft (dashed lines) looming (red) and receding (blue) stimuli for one example cortical site. Traces represent the mean response across 18 trials per condition.

ing the dynamic portion of the stimuli. However, an examination of the same signals in the frequency domain revealed strong modulation of power in the LFP in response to the dynamic portion of the stimulus. Figure $2 B$ shows time-frequency representations (spectrograms), normalized to baseline ( $500 \mathrm{~ms}$ before stimulus onset), of the LFP signals shown in Figure $2 \mathrm{~A}$. These spectro- 
grams show that looming stimuli (averaged across both loud and soft conditions) elicited a sustained increase in gamma-band (45-90 Hz) power (Fig. 2 B, top), whereas gamma-band power in response to receding stimuli (averaged across both loud and soft conditions) was not sustained (Fig. $2 B$, bottom). Figure $2 C$ shows the gamma-band-limited $(45-90 \mathrm{~Hz})$ power changes across the four different conditions separately. A two-way ANOVA with gamma power as the dependent variable and direction (looming vs receding) and intensity (loud vs soft) as factors revealed a significant main effect for direction only $(F=7.25 ; p=0.009)$ for this cortical site but not for intensity $(F=1.62 ; p=0.207$; interaction, $F=0.33 ; p=0.568)$.

Across the population of cortical sites $(n=50)$, the same overall pattern was observed. In the frequency domain, looming signals elicited sustained gamma-band responses that were not apparent in the receding sound condition (Fig. $3 A$ ). Frequencies between 25 and $45 \mathrm{~Hz}$ usually showed suppression relative to baseline, but this was not stimulus selective. No consistent modulation of frequencies $<25 \mathrm{~Hz}$ was apparent. To illustrate the degree of looming biases across the population of cortical responses, we calculated a looming preference index. Preference for looming versus receding stimuli was calculated for each cortical site by subtracting the gamma-band response elicited by the dynamic portion of the receding stimuli from the gamma-band response elicited by the dynamic portion of the looming stimuli. Looming and receding conditions in this analysis included both loud and soft stimuli. A positive value indicates preference for looming stimuli, and a negative value indicates preference for receding stimuli. Figure $3 B$ shows that most cortical sites had a preference for looming stimuli (36 of 50), and the mean preference was significantly different from zero (mean \pm SEM $=$ $0.057 \pm 0.014 ; T=4.084 ; p<0.001$ ). Across the population (Fig. $3 C)$, the two-way ANOVA revealed that the direction of change was significantly biased toward looming signals $(F=18.172 ; p<$ $0.0001)$, and there was no effect for overall intensity $(F=0.824$; $p=0.369)$ but a trend (although not significant) for an interaction $(F=2.972 ; p=0.091)$. Thus, looming stimuli elicited a stronger increase in gamma-band power in lateral belt auditory cortex than receding stimuli. Moreover, there is the suggestion that the bias for looming signals is not completely independent of the absolute intensity of the sound, which parallels findings with the perception of looming sounds by humans (Neuhoff, 1998).

\section{Looming versus receding sounds: multiunit activity}

MUA showed the same pattern as the gamma-band LFP activity. Figure $4 A$ shows firing rate in the population MUA $(n=$ 50 cortical sites), normalized to baseline (500 ms before stimulus onset), recorded from the same cortical sites reported for the LFP analyses above. Mean firing rate over the population of cortical sites is shown as a function of time in response to looming and receding stimuli. Like the LFP, looming stimuli elicited stronger spiking activity than receding stimuli during the dynamic period. This is remarkable in light of the fact that these are multiunit responses from an unbiased sample of cortical sites. Looming preference index of the population MUA shows that 30 of 50 cortical sites had a preference for looming compared with receding stimuli. The mean preference was again significantly greater than zero (mean $\pm \mathrm{SEM}=$ $0.1020 \pm 0.038 ; T=2.6120 ; p=0.0119$ ) (Fig. $4 B$ ). Figure $4 C$ shows the MUA recorded from one example cortical site, in response to the soft and loud looming and receding stimuli. Like in the gamma-band LFP response, the two-way ANOVA revealed a significant main effect of direction $(F=12.4 ; p=$
A
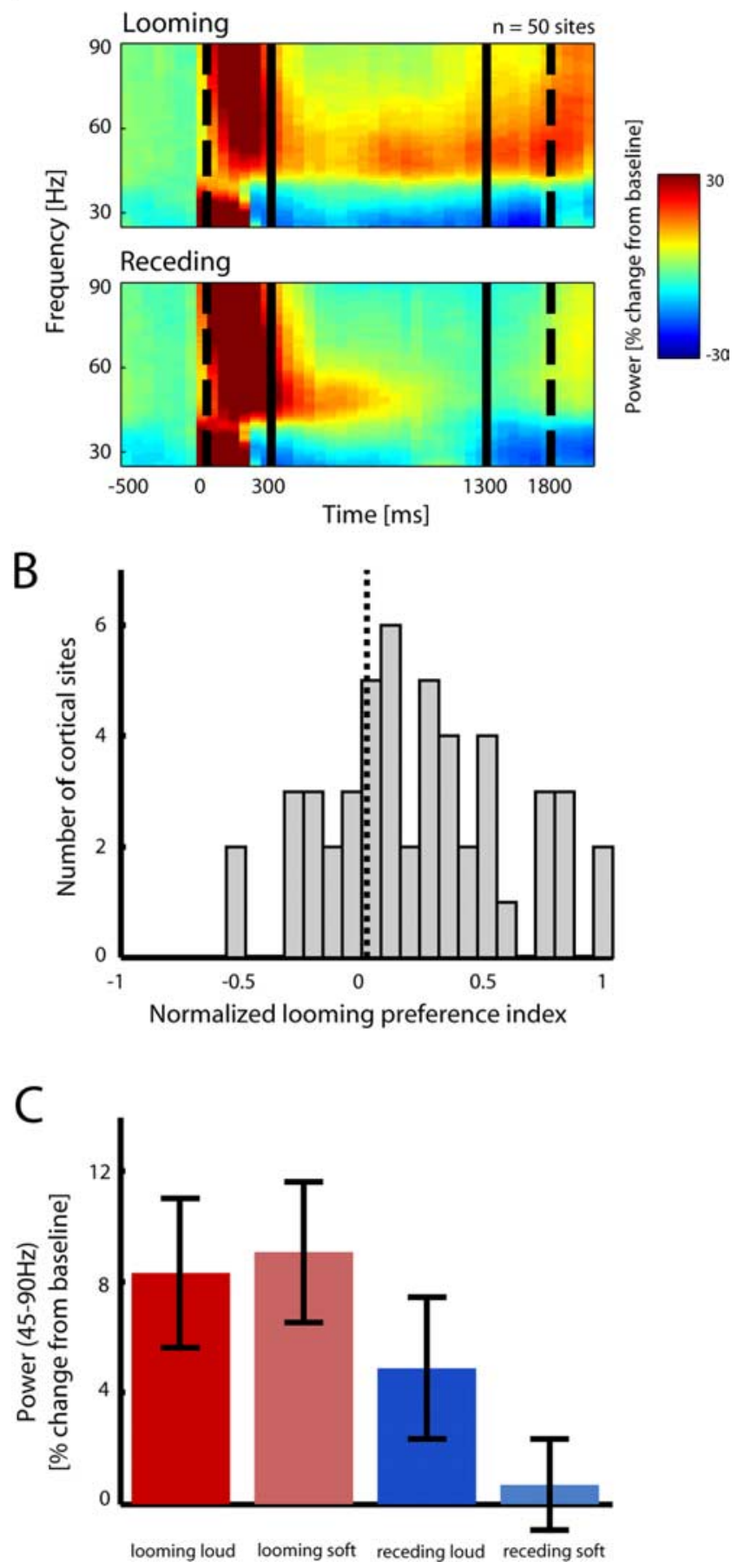

Figure 3. A, Population spectrograms in response to looming and receding stimuli. Shown are LFP spectrograms, normalized to baseline, averaged across cortical sites. Spectrograms represent the mean response in the complex tone condition across 50 cortical sites. $\boldsymbol{B}$, Normalized looming preference of the gamma-band LFP response for each cortical site. Positive values indicate preference for looming stimuli, and negative values indicate preference for receding stimuli. C, Population mean gamma-band power in response to loud and soft looming and receding stimuli. Shown is mean gamma-band power during the dynamic portion of the looming (red bars) and receding (blue bars) stimuli, relative to baseline, averaged across cortical sites. Values represent the mean response across 50 cortical sites. Error bars represent \pm 1 SEM.

$0.0008)$ but no effect of intensity $(F=0.05 ; p=0.8194$; interaction, $F=0.11 ; p=0.737)$. Across the population of MUA responses, the two-way ANOVA also revealed a significant effect of direction $(F=6.82 ; p=0.012)$ but no effect of 

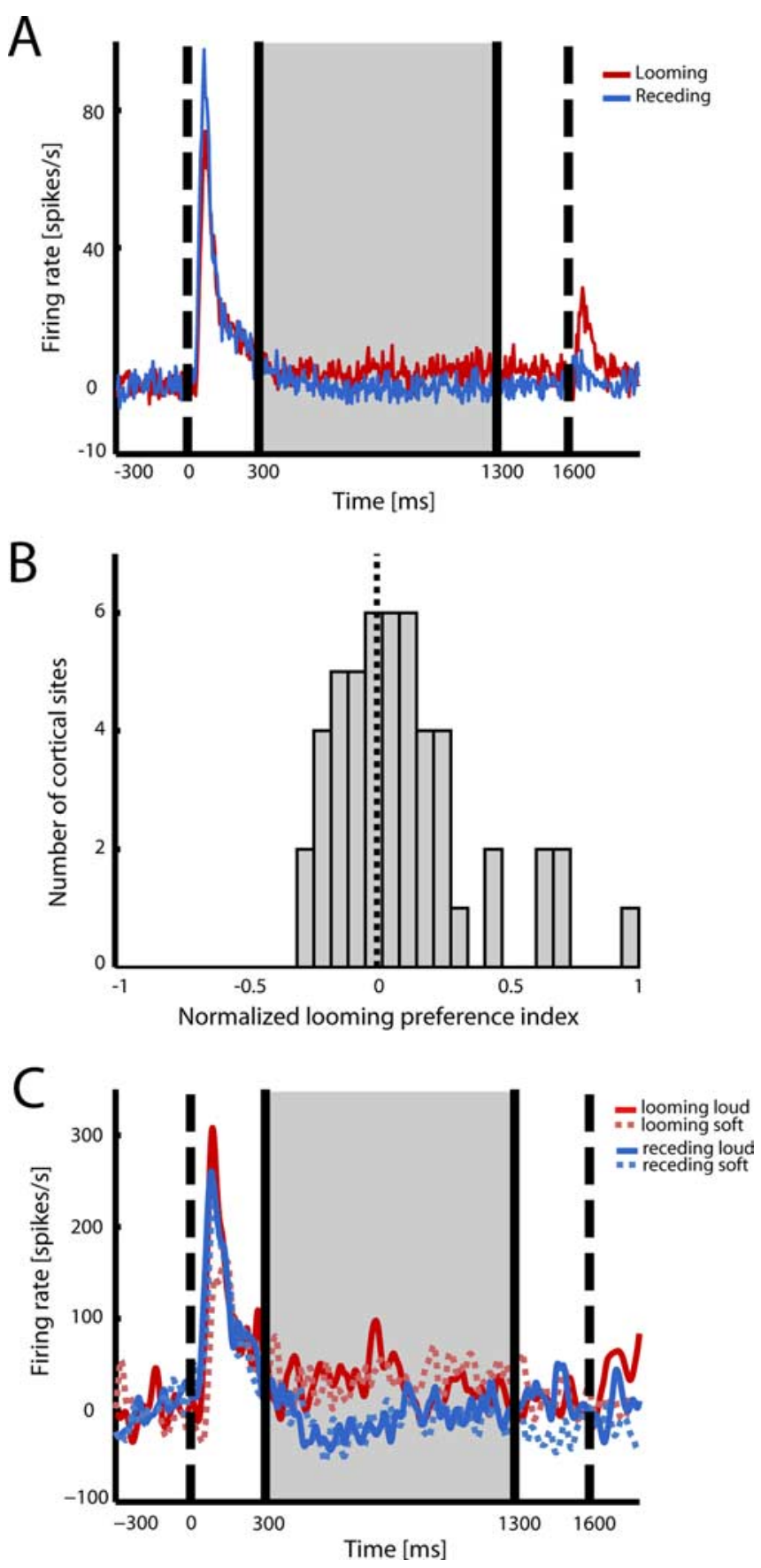

Figure 4. A, Population multiunit activity, normalized to baseline, averaged over cortical sites in response to looming (red) and receding (blue) stimuli. Traces represent the mean response across 50 cortical sites. $\boldsymbol{B}$, Normalized looming preference of the multiunit response for each cortical site. Positive values indicate preference for looming stimuli, and negative values indicate preference for receding stimuli. C, Example MUA in response to loud and soft looming and receding stimuli. Shown is multiunit activity over time, relative to baseline, in response to loud (solid lines) and soft (dashed lines) looming (red) and receding (blue) stimuli for one example cortical site. Traces represent spike density functions of one example cortical site (mean over 18 trials per condition).

intensity $(F=0.01 ; p=0.889$; interaction, $F=0.47 ; p=$ $0.496)$ on the mean firing rate during stimulus motion. In parallel with gamma-band LFP activity, these data show that MUA in the lateral belt auditory cortex has a bias for looming versus receding sounds, independent of absolute intensity.
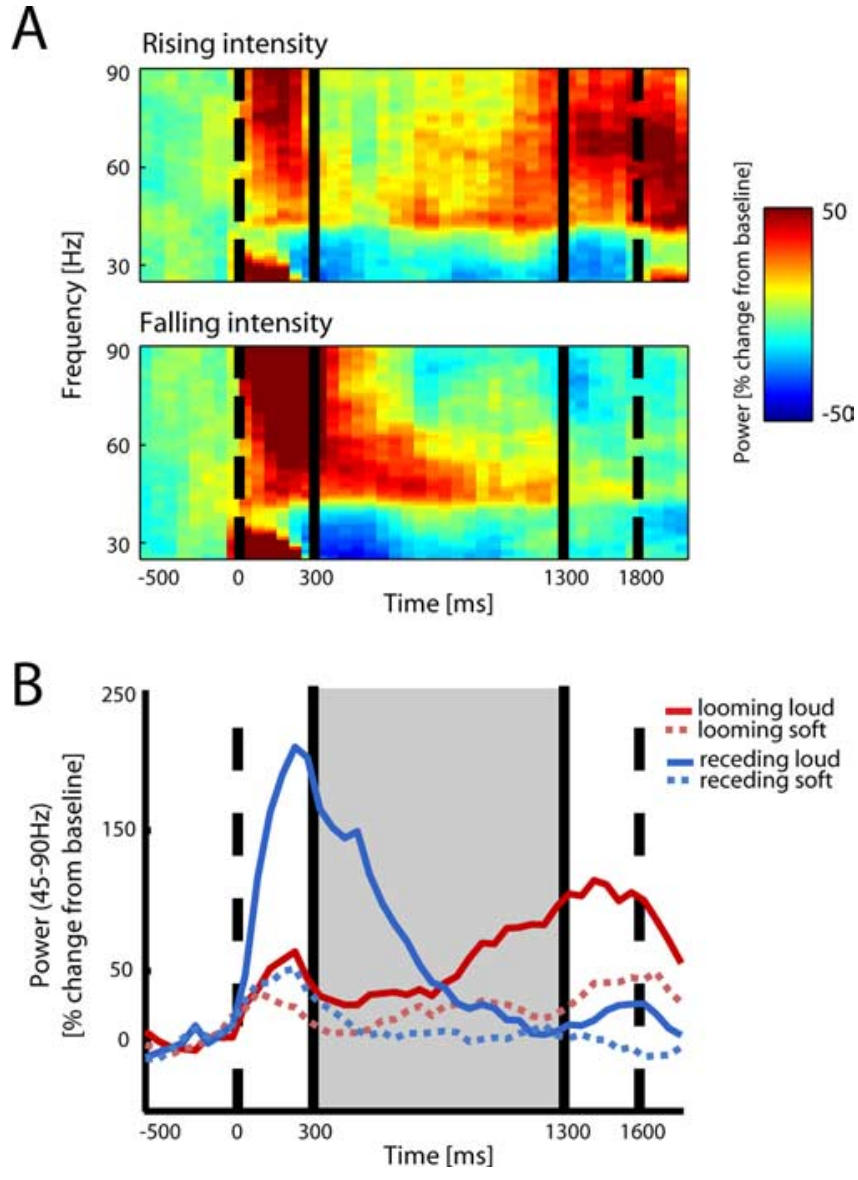

Figure 5. $A$, Spectrogram of a single cortical site's response to rising- and falling-intensity white noise stimuli. $\boldsymbol{B}$, Example gamma-band power in the LFP in response to loud and soft rising- and falling-intensity white noise stimuli. Gamma-band $(45-90 \mathrm{~Hz})$ power in the LFP over time, relative to baseline, in response to loud (solid lines) and soft (dashed lines) rising(red) and falling (blue) intensity white noise stimuli for one example cortical site. Traces represent the mean response across 18 trials per condition.

\section{Complex tones versus white noise}

The observed bias for looming sounds could be the result of adaptation to the receding sounds, which start out relatively loud and then fade out in intensity (Fay et al., 1996). To investigate this possibility, we recorded auditory cortical activity in response to rising- and falling-intensity white noise; the amplitude envelopes were identical to those used for the complex tones (Fig. 1). White noise is an ideal control stimulus for two reasons. First, because lateral belt auditory cortex responds better to white noise than to tonal sounds (Rauschecker and Tian, 2004), one would expect stronger adaptation effects in the white noise condition, because of stronger onset responses. Second, at the behavioral level, there is no perceptual bias in the perception of rising- and fallingintensity white noise sounds. Thus, if the asymmetry observed in auditory cortical activity in response to looming and receding complex tones is caused by the simple explanation outlined above, then we predicted that rising- and falling-intensity white noise are represented asymmetrically as well in auditory cortical activity.

For comparing responses to rising- versus falling-intensity stimuli, LFP responses to white noise were analyzed in the same way as the response to complex tones. We found that white noise with the exact same intensity parameters as the complex tones did not reveal an auditory cortical response bias for rising intensity. Figure 5 shows single cortical site's spectrogram in response to 
rising- and falling-intensity white noise stimuli. In contrast to the response to complex tones (Fig. $2 B$ ), gamma-band activity is not sustained throughout the duration of the stimulus, and there is no bias for rising compared with falling intensity. Figure $5 B$ shows gamma-band power changes in the LFP of one example cortical site in response to loud and soft rising- and fallingintensity white noise stimuli. A two-way ANOVA revealed no main effect of direction $(F=0.12 ; p=0.725)$ but a significant effect of intensity $(F=41.29 ; p<0.0001$; interaction, $F=2.24$; $p=0.139$ ). This example clearly illustrates two important differences between responses to white noise and complex tones. First, responses to loud stimuli were greater compared with soft stimuli. Second, there was no bias for rising-intensity stimuli.

These findings were borne out in the population-level analyses as well. Figure $6 A$ shows the population spectrogram, in which no bias for rising is apparent. On the contrary, the mean normalized rising-intensity preference index indicates a bias for falling intensity (mean \pm SEM $=-0.034 \pm 0.016 ; T=-2.13$; $p=0.0379$ ) (Fig. $6 B$ ). Figure $6 C$ shows the population gammaband power in response to the four different white noise conditions, and a two-way ANOVA revealed a significant effect of intensity $(F=26.809 ; p<0.0001)$. There was also a significant effect of direction $(F=4.514 ; p=0.039)$ but in reverse: fallingintensity stimuli elicited a slightly higher response than risingintensity stimuli. There was no significant interaction $(F=0.942$; $p=0.337)$.

MUA recorded in response to rising- and falling-intensity white noise shows a pattern very similar to the one observed in the LFP activity (data not shown). At the population level, MUA in response to the dynamic period in the four conditions also showed a significant effect of intensity $(F=7.09 ; p=0.0084)$ and no significant effect of direction $(F=2.77 ; p=0.0979$; interaction, $F=1.55 ; p=0.214$ ).

\section{Discussion}

The main finding of the present study is the existence of a bias for looming versus receding signals in auditory lateral belt cortical activity. This was true for both local field potential and multiunit activity. The observed bias for looming sounds was independent of the absolute intensity of the sound but dependent on the presence of spectral structure in the sound: white noise did not elicit the same pattern of results. These findings are strikingly parallel to the perceptual biases exhibited by both humans and monkeys.

The auditory cortical bias for looming complex tones disappears for rising-intensity white noise. The lack of a risingintensity bias in the white noise has two implications. First, it shows that the bias for rising-intensity complex tones is not the result of a general mechanism (Fay et al., 1996; Lu et al., 2001). Fay et al. (1996) argued that the initial response to a sound might deplete synapses, thereby increasing the excitability threshold of neurons. Only progressively increasing excitatory activity, in response to progressively increasing sound intensity, would then be able to move the excitation level above threshold. Consequently, rising-intensity sounds would elicit a stronger response than falling-intensity sounds. A general mechanism like this would predict that rising- and falling-intensity sounds are always represented asymmetrically in the neural activity of auditory cortex. In the present study, this was not the case for the white noise stimuli. Second, the results from the white noise condition show that responses to spectrally structured sounds can be qualitatively different from responses to unstructured sounds. Responses to rising- and falling-intensity complex tones were only dependent
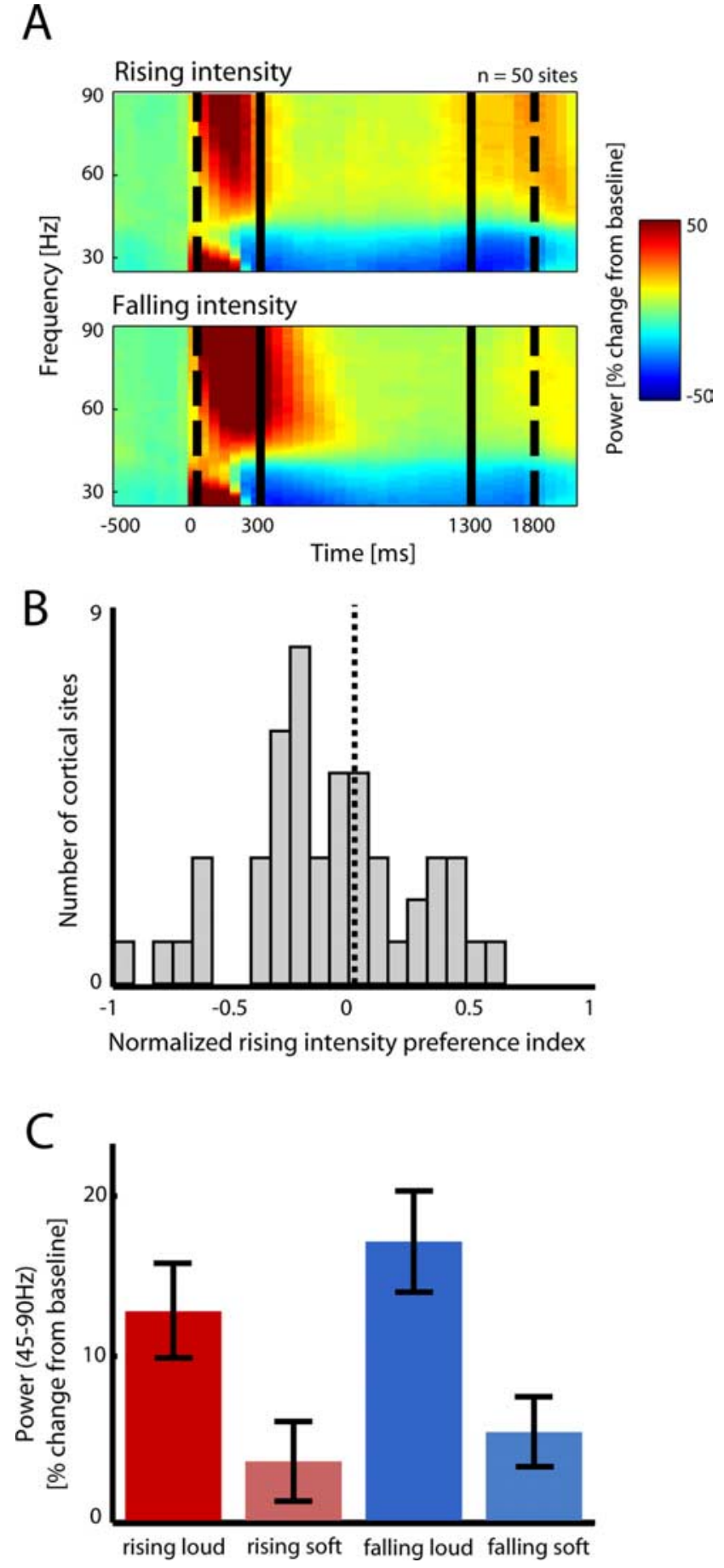

Figure 6. A, Population spectrograms of the response to rising- and falling-intensity white noise. Shown are LFP spectrograms, normalized to baseline, averaged across cortical sites. Spectrograms represent the mean response in the white noise condition across 50 cortical sites. $\boldsymbol{B}$, Normalized preference index of the LFP responses to rising- versus falling-intensity white noise. Positive values indicate preference for rising-intensity stimuli, and negative values indicate preference for falling-intensity stimuli. C, Population mean gamma-band power in response to loud and soft rising- and falling-intensity white noise stimuli. Shown is mean gamma-band power, normalized to baseline, during the dynamic portion of the rising- (red bars) and falling (blue bars)-intensity stimuli, relative to baseline, averaged across cortical sites. Values represent the mean response across 50 cortical sites. Error bars represent \pm 1 SEM.

on the direction of intensity change, with no effect of absolute intensity. Conversely, responses to rising- and falling-intensity white noise were mostly dependent on the absolute intensity of the sound, with loud sounds eliciting greater responses than soft 
sounds. Direction of intensity change had only a modest effect (and in the opposite direction of the complex tone responses) on the response magnitude.

The observed auditory cortical bias for rising-intensity complex tones closely parallels results from behavioral studies in human adults and infants (Small, 1977; Morrongiello et al., 1991; Reinhardt-Rutland, 1996a,b; Neuhoff, 1998; Freiberg et al., 2001) and monkeys (Ghazanfar et al., 2002). Indeed, a subset of these experiments have shown that the perceptual bias for looming sounds is dependent on the presence of spectral structure in the sound; white noise stimuli do not elicit any bias (Neuhoff, 1998; Ghazanfar et al., 2002; Maier et al., 2004). It has been argued that the underlying reason for this spectral dependency is that naturally occurring sound sources always produce sounds that contain some spectral structure, whereas white noise is associated with more dispersed phenomena such as wind or rain (Bregman, 1990). Thus, white noise is not likely to be associated with a single approaching object, and there would be no adaptive reason for a looming bias toward such sounds.

It is important to note that the responses to dynamic, longduration sounds observed in the present study could not have been predicted on the basis of simple intensity tuning functions, obtained from responses to short, static sounds. Such conventional intensity tuning functions can be monotonic (i.e., the neural response increases or decreases linearly with increasing sound intensity) or they can be nonlinear (nonmonotonic) (Pfingst and O'Connor, 1981; Recanzone et al., 2000a). In the present study, because the absolute intensity of rising- and falling-intensity sounds was equal, such tuning functions would not predict a difference in the response between these two conditions. Moreover, on the basis of monotonic tuning functions, one would predict a difference in the response between loud and soft auditory stimuli. Neither of these predictions was true for the activity elicited in response to dynamic complex tone stimuli.

Few studies have previously looked at the neural representation of rising- versus falling-intensity sounds. Lu et al. (2001) found a bias in single-unit activity for rising- versus fallingintensity sounds in the auditory cortex of the marmoset monkey. However, their stimuli were extremely short ( $25 \mathrm{~ms}$ ) and do not relate, perceptually or physically, to the dynamic distance of a sound source. In anesthetized cats, Stumpf et al. (1992) showed a bias for rising-versus falling-intensity pure tones in the singleunit activity in primary auditory cortex. However, the dynamic interval of their stimuli was also relatively short $(250 \mathrm{~ms})$ compared with the present study, and responses did not appear to be sustained. Moreover, because they adjusted the absolute intensity of the stimuli to the intensity threshold of each single neuron, it was impossible to test for the relative contribution of absolute intensity to the firing rate. In an initial study using functional magnetic resonance imaging in humans, Seifritz et al. (2002) showed that looming sounds activated areas involved in auditory motion processing more strongly than receding sounds. In contrast to the present results, auditory cortex itself was not found to be differentially activated by looming and receding sounds. This is probably because of the block design used in that study, which may have led to habituation effects. Indeed, a more recent study by the same group using the same stimuli and an event-related design demonstrates that looming sounds elicit greater activity in auditory cortical areas in the temporal plane (but outside Heschl's gyrus, or primary auditory cortex) than do receding sounds (Bach et al., 2007), consistent with our present results. However, it is not clear whether the lateral belt area in our study is homologous to the temporal plane activation seen by Bach et al. (2007).
This is in large part because of the lack of comparative anatomical data examining the similarities and differences between the auditory cortices [beyond the core region (Hackett et al., 2001)] of monkeys, apes, and humans.

The selective modulation of the LFP signals in the present study was confined to the gamma band. The raw LFP has a $1 / f$ structure: most power is contained in low frequencies and falls off toward higher frequencies. Therefore, selective but lowamplitude, high (gamma)-frequency responses can be masked by nonselective, high-amplitude low frequencies in the raw LFP. This underscores the importance of considering band-limited power in the LFP, as opposed to the broadband, raw LFP. Thus, our findings are reminiscent of results in other domains of sensory and mnemonic processing. In monkeys, gamma-band power has previously been shown to be selectively modulated by visual stimuli in the primary visual cortex and middle temporal area (Henrie and Shapley, 2005; Liu and Newsome, 2006), by pure tones in A1 (Brosch et al., 2002), and by stimulus-driven cognitive functions, such as attention in area V4 (Fries et al., 2001) and working memory in the posterior parietal cortex (Pesaran et al., 2002).

In summary, the biases observed in the present study are dependent on both the dynamic nature of the stimuli and the use of naturalistic, spectrally structured sounds. These findings reveal two important properties of auditory cortical processing. First, responses to dynamic stimuli cannot always be predicted on the basis of responses to static sounds. Second, responses to spectrally structured sounds can be qualitatively different from responses to unstructured sounds. Our results also show a close parallel between activity in lateral belt auditory cortex and natural behavioral biases, previously observed in primates in response to auditory looming signals. The asymmetric neural processing of looming versus receding sounds in lateral belt auditory cortex possibly reflects an adaptive mechanism that creates a margin of safety when dealing with rapidly approaching danger. Thus, the lateral belt cortex represents one node in a network adapted to respond to warning cues (Bremmer et al., 2001; Seifritz et al., 2002; Cooke and Graziano, 2004; Bach et al., 2007).

\section{References}

Bach DR, Schachinger H, Neuhoff JG, Esposito F, Di Salle F, Lehmann C, Herdener M, Scheffler K, Seifritz E (2007) Rising sound intensity as an intrinsic warning cue. Cereb Cortex, in press.

Barbour DL, Wang X (2003) Contrast tuning in auditory cortex. Science 299:1073-1075.

Bregman AS (1990) Auditory scene analysis: the perceptual organization of sound. Cambridge, MA: MIT.

Bremmer F, Schlack A, Shah NJ, Zafiris O, Kubischik M, Hoffmann KP, Zilles K, Fink GR (2001) Polymodal motion processing in posterior parietal and premotor cortex: a human fMRI study strongly implies equivalencies between humans and monkeys. Neuron 29:287-296.

Brosch M, Budinger E, Scheich H (2002) Stimulus-related gamma oscillations in primate auditory cortex. J Neurophysiol 87:2715-2725.

Cooke DF, Graziano MS (2004) Sensorimotor integration in the precentral gyrus: polysensory neurons and defensive movements. J Neurophysiol 91:1648-1660.

Dean I, Harper NS, McAlpine D (2005) Neuronal population coding of sound level adapts to stimulus statistics. Nat Neurosci 8:1684-1689.

Fay RR, Chronopoulos M, Patterson RD (1996) The sound of a sinusoid: perception and neural representations in the goldfish (Cassius auratus). Audit Neurosci 2:377-392.

Freiberg K, Tually K, Crassini B (2001) Use of an auditory looming task to test infants' sensitivity to sound pressure level as an auditory distance cue. Br J Dev Psychol 19:1-10.

Fries P, Reynolds JH, Rorie AE, Desimone R (2001) Modulation of oscillatory neuronal synchronization by selective visual attention. Science 291:1560-1563. 
Gabbiani F, Krapp HG, Koch C, Laurent G (2002) Multiplicative computation in a visual neuron sensitive to looming. Nature 420:320-324.

Garcia-Lazaro JA, Ahmed B, Schnupp JWH (2006) Tuning to natural stimulus dynamics in primary auditory cortex. Curr Biol 16:264-271.

Ghazanfar AA, Neuhoff JG, Logothetis NK (2002) Auditory looming perception in rhesus monkeys. Proc Natl Acad Sci USA 99:15755-15757.

Ghazanfar AA, Maier JX, Hoffman KL, Logothetis NK (2005) Multisensory integration of dynamic faces and voices in rhesus monkey auditory cortex. J Neurosci 25:5004-5012.

Hackett TA, Stepniewska I, Kaas JH (1998) Subdivisions of auditory cortex and ipsilateral cortical connections of the parabelt auditory cortex in macaque monkeys. J Comp Neurol 394:475-495.

Hackett TA, Preuss TM, Kaas JH (2001) Architectonic identification of the core region in auditory cortex of macaques, chimpanzees, and humans. J Comp Neurol 441:197-222.

Henrie JA, Shapley R (2005) LFP power spectra in V1 cortex: the graded effect of stimulus contrast. J Neurophysiol 94:479-490.

Lehmann C, Herdener M, Esposito F, Hubl D, di Salle F, Scheffler K, Bach DR, Federspiel A, Kretz R, Dierks T, Seifritz E (2006) Differential patterns of multisensory interactions in core and belt areas of human auditory cortex. NeuroImage 31:294-300.

Liu J, Newsome WT (2006) Local field potential in cortical area MT: Stimulus tuning and behavioral correlations. J Neurosci 26:7779-7790.

Lu T, Liang L, Wang X (2001) Neural representations of temporally asymmetric stimuli in the auditory cortex of awake primates. J Neurophysiol 85:2364-2380.

Lufti RA, Wang W (1999) Correlational analysis of acoustic cues for the discrimination of auditory motion. J Acoust Soc Am 106:919-928.

Maier JX, Neuhoff JG, Logothetis NK, Ghazanfar AA (2004) Multisensory integration of looming signals by rhesus monkeys. Neuron 43:177-181.

Middlebrooks JC, Pettigrew JD (1981) Functional classes of neurons in primary auditory cortex of the cat distinguished by sensitivity to sound location. J Neurosci 1:107-120.

Mitra PP, Pesaran B (1999) Analysis of dynamic brain imaging data. Biophys J 76:691-708.

Morel A, Garraghty PE, Kaas JH (1993) Tonotopic organization, architectonic fields, and connections of auditory-cortex in macaque monkeys. J Comp Neurol 335:437-459.

Morrongiello BA, Hewitt KL, Gotowiec A (1991) Infants discrimination of relative distance in the auditory modality-approaching versus receding sound sources. Infant Behav Dev 14:187-208.

Nelken I, Rotman Y, Bar Yosef O (1999) Responses of auditory cortex neurons to structural features of natural sounds. Nature 397:154-157.

Neuhoff JG (1998) Perceptual bias for rising tones. Nature 395:123-124.

Neuhoff JG (2001) An adaptive bias in the perception of looming auditory motion. Ecol Psychol 13:87-110.

Palanca BJ, DeAngelis GC (2005) Does neural synchrony underlie visual feature grouping? Neuron 46:333-346.

Pesaran B, Pezaris JS, Sahani M, Mitra PP, Andersen RA (2002) Temporal structure in neuronal activity during working memory in macaque parietal cortex. Nat Neurosci 5:805-811.

Pfingst BE, O'Connor TA (1980) Vertical stereotaxic approach to auditorycortex in the unanesthetized monkey. J Neurosci Methods 2:33-45.

Pfingst BE, O'Connor TA (1981) Characteristics of neurons in auditory- cortex of monkeys performing a simple auditory task. J Neurophysiol 45:16-34.

Raghavachari S, Kahana MJ, Rizzuto DS, Caplan JB, Kirschen MP, Bourgeois B, Madsen JR, Lisman JE (2001) Gating of human theta oscillations by a working memory task. J Neurosci 21:3175-3183.

Rauschecker JP, Tian B (2004) Processing of band-passed noise in the lateral auditory belt cortex of the rhesus monkey. J Neurophysiol 91:2578-2589.

Rauschecker JP, Tian B, Hauser M (1995) Processing of complex sounds in the macaque nonprimary auditory-cortex. Science 268:111-114.

Recanzone GH (2000) Response profiles of auditory cortical neurons to tones and noise in behaving macaque monkeys. Hear Res 150:104-118.

Recanzone GH, Guard DC, Phan ML (2000a) Frequency and intensity response properties of single neurons in the auditory cortex of the behaving macaque monkey. J Neurophysiol 83:2315-2331.

Recanzone GH, Guard DC, Phan ML, Su TK (2000b) Correlation between the activity of single auditory cortical neurons and sound-localization behavior in the macaque monkey. J Neurophysiol 83:2723-2739.

Reinhardt-Rutland AH (1996a) Asymmetrical perception of changing intensity in short tonal stimuli: duration of stimulus. J Gen Psychol 123:115-122.

Reinhardt-Rutland AH (1996b) The illusion of increasing loudness in brief steady tones: variation with carrier frequency. J Gen Psychol 123:325-332.

Robinson DA (1963) A method of measuring eye movement using a scleral search coil in a magnetic field. IEEE Trans Biomed Eng 10:137-145.

Rosenblum LD, Carello C, Pastore RE (1987) Relative effectiveness of three stimulus variables for locating a moving sound source. Perception 16:175-186.

Scherberger H, Jarvis MR, Andersen RA (2005) Cortical local field potential encodes movement intentions in the posterior parietal cortex. Neuron 46:347-354.

Schiff W (1965) Perception of impending collision—a study of visually directed avoidant behavior. Psychol Monogr 79:1-26.

Schiff W, Oldak R (1990) Accuracy of judging time to arrival-effects of modality, trajectory, and gender. J Exp Psychol Hum Percept Perform 16:303-316.

Schiff W, Caviness JA, Gibson JJ (1962) Persistent fear responses in rhesus monkeys to the optical stimulus of "looming." Science 136:982-983.

Seifritz E, Neuhoff JG, Bilecen D, Scheffler K, Mustovic H, Schachinger H, Elefante R, Di Salle F (2002) Neural processing of auditory looming in the human brain. Curr Biol 12:2147-2151.

Small AM (1977) Loudness perception of signals of monotonically changing sound pressure. J Acoust Soc Am 61:1293-1297.

Stumpf E, Toronchuk JM, Cynader MS (1992) Neurons in cat primary auditory cortex sensitive to correlates of auditory motion in 3-dimensional space. Exp Brain Res 88:158-168.

Sun HJ, Frost DO (1998) Computation of different optical variables of looming objects in pigeon nucleus rotundus neurons. Nat Neurosci 1:296-303.

Super H, Roelfsema PR (2005) Chronic multiunit recordings in behaving animals: advantages and limitations. Prog Brain Res 147:263-282.

Wang X, Lu T, Snider RK, Liang L (2005) Sustained firing in auditory cortex evoked by preferred stimuli. Nature 435:341-346.

Zakarauskas P, Cynader MS (1991) Aural intensity for a moving sound source. Hear Res 52:233-244. 